\title{
Necesidades de apoyos en adultos con discapacidad intelectual institucionalizados: estudio en el contexto chileno*
}

\author{
Supports Needs for Institutionalized Adults with Intellectual \\ Disability: a Study Within the Chilean Context
}

\author{
Vanessa Vega Córdova** \\ Pontificia Universidad Católica \\ de Valparaíso, Chile \\ Cristina Jenaro Río \\ Universidad de Salamanca, España \\ Noelia Flores Robaina \\ Universidad de Salamanca, España \\ Maribel Cruz Ortiz \\ Universidad de Salamanca, España \\ Universidad Autónoma de San Luis Potosí, \\ México \\ Andrés Lerdo de Tejada \\ Fundación Coanil, Chile \\ Recibido: 24 de febrero de 2012 \\ Revisado: 24 de abril de 2012 \\ Aceptado: 17 de julio de 2012
}

\section{Resumen}

El presente estudio analiza las necesidades de apoyos de una muestra de 285 adultos con discapacidad intelectual institucionalizados. El instrumento empleado ha sido la Escala de Intensidad de Apoyos, SIS (Verdugo, Arias \& Ibañez, 2007). Los resultados revelaron la existencia de bajas necesidades de apoyos en cuanto al perfil general de la muestra, si las mayores necesidades están en las dimensiones de Aprendizaje a lo largo de la vida y la dimensión con menores necesidades de apoyos es Actividades de la vida en el hogar. Los años institucionalizados o la edad no se encontraron asociados con el nivel de las necesidades de apoyos, pero sí la severidad de la discapacidad y el género. Estos resultados avalan la validez transcultural de la SIS y su utilidad para planificar servicios orientados a mejorar la vida de la población institucionalizada con discapacidad intelectual.

Palabras clave: Necesidades de apoyos, evaluación, discapacidad intelectual, institucionalización.

Artículo de Investigación. El trabajo es parte del desarrollo de una tesis doctoral, defendida en la Universidad de Salamanca, en el programa de Doctorado de Investigación sobre Discapacidad.

** Correspondencia: Vanessa Vega Córdova. Pontificia Universidad Católica de Valparaíso (Chile). Dirección postal: Avda. El Bosque 1290, Sausalito, Viña del Mar, Chile. Correo electrónico: vanejuay@gmail.com; Cristina Jenaro Río, Universidad de Salamanca España. Facultad de Psicología. Universidad de Salamanca. Avda. de la Merced, 109-131, 37005-Salamanca (España). Correo electrónico: crisje@usal.es. Noelia Flores Robaina, Universidad de Salamanca, España. Correo electrónico: nrobaina@usal.es, Maribel Cruz Ortiz Universidad de Salamanca, España. Universidad Autónoma de San Luis Potosí, México. Correo electrónico: redazul@hotmail.com. Andrés Lerdo de Tejada, Fundación Coanil, Chile. Correo electrónico: andresldt@coanil.cl

Agradecimiento: los autores agradecen a la Fundación Coanil por su apoyo, facilitación del trabajo de campo y valiosas sugerencias para la investigación. 


\section{Abstract}

This study examines the support needs of a sample of 285 institutionalized adults with intellectual disabilities. The instrument used was the Supports Intensity Scale SIS. The results revealed the existence of low support needs, with more elevated needs in lifelong learning activities and less in home living domains. Years institutionalized and age were not associated with the level of support needs, but the severity of the disability and the gender were. These results support the cross-cultural validity of the SIS and its usefulness for planning services oriented to improving the lives of institutionalized population with intellectual disabilities.

Keywords: Support needs, assessment, intellectual disability, institutionalization.

\section{Introducción}

En la actualidad el concepto de discapacidad intelectual ha experimentado grandes cambios, acentuándose la perspectiva socio-ecológica, la importancia de la capacitación personal, la autodeterminación, los derechos y resultados personales (Thompson, et ál., 2009). Del mismo modo, cada vez es más generalizada la toma de conciencia sobre los efectos negativos de la discriminación y falta de oportunidades para las personas con discapacidad (Carter, Owens, Trainor, Sun \& Swedeen, 2009).

Entender la concepción actual de la discapacidad intelectual requiere comprender el modelo de los apoyos. Este se dirige a evaluar las discrepancias entre las capacidades y habilidades de una persona y los requerimientos y demandas del ambiente. Entendemos por apoyos todos aquellos recursos y estrategias que favorecen el acceso a la información y relaciones en contextos integrados y que incrementan la interdependencia/independencia, productividad, integración en la comunidad y satisfacción, entre otros resultados personales (Luckasson et ál., 1992; AARM, 2002; AAIDD, 2010; Verdugo, Arias, Ibañez \& Schalock, 2010).

Los apoyos garantizan la igualdad, aumentan la integración y mejoran el crecimiento y desarrollo personal; además se pueden evaluar de acuerdo con sus resultados (van Loon, Claes, Vandevelde, Van Hove \& Schalock, 2010). El empleo con apoyo o la vivienda con apoyo, son ejemplos de modelos de provisión de servicios que pueden ser evaluados en cuanto a su eficacia y eficiencia para lograr mejoras en la vida de las personas con discapacidad (McCormack \& Farrell, 2009). La naturaleza de los apoyos es muy variada y puede partir del propio sujeto, su familia y amigos, abarcando desde apoyos informales (apoyos naturales) hasta los apoyos que ofrecen los servicios especializados (apoyos profesionales) (Luckasson et ál., 1992; AARM, 2002; AAIDD, 2010).

La concepción de las necesidades de apoyos se basa en la premisa de que el funcionamiento humano se ve influenciado por el grado de congruencia entre la capacidad individual y los ambientes donde las personas se desenvuelven (Thompson, et ál., 2009; van Loon, et ál., 2010). De entre estos diferentes ambientes se destacan donde las personas con discapacidad intelectual viven. En este sentido, los apoyos residenciales han variado a lo largo de los años en cuanto a dimensiones políticas, filosóficas y físicas. Estos cambios han producido diferentes consecuencias en la vida de las personas con discapacidad (Lakin \& Stancliffe, 2007; Mansell, Beable-Brown, Skidmire, Welton \& Hutchinson; 2006, Stancliffe, Emerson \& Lakin, 2000). Dichos estudios coinciden en la necesidad de modificar los apoyos proporcionados ante cambios en el contexto, para garantizar el equilibrio entre demandas y recursos.

Si bien en el contexto internacional contamos con numerosos estudios al respecto, en el contexto hispanohablante se cuenta con estudios acerca de la calidad de vida de personas con discapacidad (Mora, Córdoba, Bedoya \& Verdugo, 2007) pero es necesario potenciar las investigaciones relacionadas con la evaluación de los apoyos requeridos 
y utilizados con esta población. Si bien contamos para ello con instrumentos como la Escala de Intensidad de Apoyos, SIS (Verdugo, Arias \& Ibañez, 2007) adaptada al castellano de la versión original Supports Intensity Scale (Thompson, et ál., 2004). La escala SIS proporciona información para identificar perfiles e intensidad de necesidades de apoyo. Permite también estructurar y evaluar el desarrollo de planes personalizados de apoyo para personas con discapacidad. Algunos estudios en el contexto latino avalan su utilidad, al menos en población mexicana con enfermedad mental (Cruz, Jenaro, Pérez \& Flores, 2010; Jenaro, Cruz, Pérez, Flores, \& Vega, 2011) o en estudios preliminares con población chilena con discapacidad intelectual (Vega, Jenaro, Morillo, Cruz \& Flores, 2011). Son, sin embargo, muy escasos los estudios realizados en Latinoamérica por lo que profundizar en esta evaluación y en el impacto de factores contextuales y personales en los apoyos proporcionados a esta población, contribuirán a mejorar la provisión de servicios.

Así pues, en el presente estudio creemos poder contribuir a contrastar la utilidad de la SIS en nuestro contexto. Esto es, demostrar que es una escala fiable y válida para ser utilizada con población chilena con discapacidad intelectual. Esperamos además identificar qué apoyos requieren las personas con discapacidad intelectual que viven en centros residenciales permanentes; como también conocer qué variables, tanto personales como contextuales, pueden estar asociadas a estas necesidades de apoyos. Tres hipótesis se plantean en el estudio, a saber: (1) las necesidades de apoyos serán elevadas; (2) las necesidades de apoyo se encontrarán positiva y significativamente asociadas con la severidad de la discapacidad y con los años institucionalizados y (3) no existirá asociación entre las necesidades de apoyo y el género o la edad.

\section{Método}

\section{Participantes}

Los participantes fueron 285 personas con discapacidad intelectual mayores de 18 años, resi- dentes en centros de la fundación Coanil de las regiones Quinta y Metropolitana. El $49.5 \%$ eran hombres y el $50.5 \%$ mujeres. Según el grado de discapacidad intelectual, la mayoría (55.4\%) presentaba una discapacidad severa, seguido de un $17.9 \%$ que presentaba discapacidad moderada, un $16.5 \%$ discapacidad profunda y un $9.8 \%$ discapacidad leve, según criterios DSM-IV. La edad promedio de los participantes era de 29.3 años, con un rango entre 18 y 51 años. En cuanto a los años que llevaban viviendo en la institución, el promedio era de 14.4 con un rango entre uno y 32 años.

Las escalas fueron respondidas por 83 informantes, responsables de valorar entre dos y cuatro usuarios. Para cada caso se eligió a aquel profesional con mayor conocimiento del residente en diversos contextos. Un $21.1 \%$ de los informantes eran trabajadoras sociales; un $22.5 \%$ eran profesores de educación especial y un $25.5 \%$ eran personal de apoyo directo. Por término medio los profesionales conocían a los usuarios desde hacía 8 años (DT=8.5).

\section{Instrumento}

Se utilizó la Escala de Intensidad de Apoyos SIS (adaptación española de Verdugo, Arias \& Ibáñez, 2007) que evalúa los apoyos requeridos por una persona con discapacidad para participar en las actividades de la vida diaria. La SIS consta de tres secciones. La Sección 1 incluye la Escala de necesidades de apoyos e incluye a su vez 49 actividades agrupadas en 6 subescalas: Vida en el hogar, Vida en la comunidad, Aprendizaje a lo largo de la vida, Empleo, Salud y seguridad $y$, Social. Las puntuaciones resultantes permiten obtener una puntuación estándar compuesta o Índice de Necesidades de Apoyo. La Sección 2 contiene la Escala suplementaria de protección y defensa, comprende 8 ítems que valoran la capacidad del usuario para defenderse, tomar decisiones o ejercer responsabilidades civiles, entre otros indicadores. La Sección 3 incluye la Escala de Necesidades Excepcionales de Apoyo Médico y Conductual y valora la posible existencia de condiciones adicionales que requieran mayores niveles de apoyos. 
Las necesidades de apoyos en las dos primeras secciones se evalúan en una escala tipo Likert (de 0 a 4) empleando para ello tres medidas diferentes: a) frecuencia del apoyo, b) tiempo diario de apoyo y c) tipo o intensidad de apoyo. En el presente trabajo, el análisis de la consistencia interna de los 49 ítems fue de $\alpha=.98$ para la frecuencia de los apoyos, de $\alpha=.99$ para el tiempo de apoyo y de $\alpha=.99$ para el tipo de apoyos. Los niveles de fiabilidad para las diferentes subescalas mostraron un rango entre .876 y .984 . Estos datos fueron ligeramente superiores a los obtenidos con población con enfermedad mental (Cruz et ál., 2010; Jenaro et ál., 2011). Fueron por otro lado ampliamente similares a los obtenidos tanto en los estudios originales con la escala (Thompson et ál., 2004) como en la validación española (Verdugo et ál., 2007). En ambos casos los valores fueron superiores a .90 para las diferentes subescalas.

\section{Procedimiento}

La investigación es de carácter transversal descriptivo y diseño ex post facto, se ha llevado a cabo en siete centros pertenecientes a la Fundación Coanil. Coanil es la fundación privada de servicios residenciales con más antiguedad de Chile.

La presente ha seguido un proceso de tres fases. La primera es el contacto con la Fundación Coanil, quienes aprobaron el protocolo de evaluación y con quienes se firmó un convenio de colaboración. La segunda consistió en la realización de charlas informativas al personal de la fundación y programación del trabajo de campo con cada uno de los hogares. La tercera fase implicó la recogida de datos a través de entrevistas al personal de los hogares y se llevó a cabo de agosto a diciembre de 2008. Las entrevistas fueron realizadas por la responsable principal del estudio, junto con alumnos de quinto año de la carrera de Educación Diferencial de la Pontificia Universidad Católica de Valparaíso, quienes fueron previamente entrenados. Los informantes de los diferentes centros residenciales consistieron en participar tras garantizárseles la confidencialidad de la información recogida.

\section{Análisis de datos}

Hemos empleado estadísticos de los tests para valorar las propiedades psicométricas de las pruebas empleadas. Concretamente, hemos analizado la consistencia interna de la escala y sus diferentes secciones mediante el estadístico Alfa de Cronbach. Respecto a la validez, esperamos ofrecer evidencias de la validez de constructo siguiendo los análisis de los autores de la SIS y de su validación al castellano, esto es: (1) mediante el cálculo de la asociación entre las puntuaciones en la SIS y la edad y el género (esperamos una ausencia de asociación entre dichas variables). (2) Mediante el análisis de la esperada asociación entre las puntuaciones en la SIS, y la severidad de la discapacidad y años institucionalizados.

Asimismo, para dar respuesta a nuestros objetivos y realizar el contraste de hipótesis hemos empleado estadísticos descriptivos e inferenciales. Las pruebas para el contraste de hipótesis empleadas han sido bivariadas (correlaciones, análisis de varianza) y multivariadas (análisis MLG multivariante que proporciona un análisis de varianza para múltiples variables dependientes). De este modo, si una prueba multivariada era significativa, procedíamos a emplear los contrastes univariados; en caso contrario no continuábamos con los análisis. Ello reduce la probabilidad de errores debido a la realización de múltiples comparaciones (Cooley \& Lohnes, 1971). Empleamos además pruebas de contraste post hoc (Scheffé y Duncan) para determinar en su caso los grupos que diferían significativamente de los demás.

\section{Resultados}

Presentamos en primer lugar los promedios, rangos y dispersión de las puntuaciones en la SIS. Esta evaluación equivale a lo que Thompson et ál., (2009) denominan "necesidades normativas". En la tabla 1 se ofrecen las puntuaciones promedio obtenidas en las diferentes actividades de la Sección A de la SIS. Se puede apreciar cómo las mayores necesidades de apoyo surgen en las Actividades de aprendizaje a lo largo de la vida. Por otro lado, las menores necesidades de apoyo se relacionan con las Actividades de vida en el hogar. 
Tabla 1.

Puntuaciones media y desviación típica en la parte A (necesidades de apoyo) de la Escala SIS

\begin{tabular}{ccccc}
\hline & Mínimo & Máximo & M & DT \\
\hline AV & 0 & 104 & 63.96 & 23.10 \\
VC & 0 & 91 & 53.54 & 22.55 \\
AE & 0 & 87 & 52.35 & 20.95 \\
SS & 0 & 93 & 51.26 & 20.13 \\
AS & 0 & 93 & 45.66 & 24.06 \\
VH & 0 & 92 & 40.61 & 22.41 \\
\hline
\end{tabular}

Nota: $\mathrm{AV}=$ Actividades de aprendizaje a lo largo de la vida; VC=Actividades de la vida en la comunidad; $A E=$ Actividades de empleo; $S S=$ Actividades de salud y seguridad; $A S=$ Actividades sociales; $\mathrm{VH}=$ Actividades de la vida en el hogar.

A continuación y en relación con las necesidades de apoyos detectadas a través de la Sección 2: Escala suplementaria de protección y defensa, de la SIS, los resultados se resumen en la Tabla 2. Se aprecia la existencia de mayores necesidades de apoyo relacionadas con Manejar dinero y finanzas personales y menores necesidades relacionadas con Defender a otros.

Tabla 2.

Puntuaciones media y desviación típica en la Sección 2 de la Escala SIS (actividades de protección y defensa)

\begin{tabular}{lcccc}
\hline Actividades de protección y defensa & Mínimo & Máximo & M & DT \\
\hline 2.1 Defenderse ante los demás & 0 & 11 & 5.09 & 3.55 \\
2.2 Manejar dinero y finanzas personales & 0 & 12 & 8.31 & 2.62 \\
2.3 Protegerse a sí mismo de la explotación & 0 & 12 & 7.24 & 3.47 \\
2.4 Ejercer responsabilidades legales & 0 & 12 & 7.89 & 2.83 \\
2.5 Pertenecer y participar en organizaciones de autodefensa o autoayuda & 0 & 12 & 7.66 & 2.94 \\
2.6 Obtener servicios legales & 0 & 12 & 8.06 & 2.73 \\
2.7 Hacer elecciones y tomar decisiones & 0 & 12 & 6.50 & 3.41 \\
2.8 Defender a otros & 0 & 11 & 5.91 & 3.65 \\
\hline
\end{tabular}

En cuanto a los resultados relativos a la Sección 3: Necesidades excepcionales de apoyo médico y conductual, las puntuaciones más elevadas se obtuvieron en las variables: Servicios de terapia (Media $=0.81$; DT $=.82$ ) y Otros servicios (Media= 0.43 ; DT=.74). Con respecto a las necesidades de apoyo conductual, las mayores necesidades se relacionan con la Prevención de rabietas o estallidos emocionales (Media=0.93; DT=.76) y con el Mantenimiento de los tratamientos de salud mental (Media= 0.87; DT=.82).

Respecto al Índice de Necesidades de Apoyo, el $82.8 \%(N=236)$ de la muestra presentan bajos ni- veles de apoyo, el 14.7\% ( $\mathrm{N}=42)$ presentan necesidades medias y el $1.4 \%(\mathrm{~N}=4)$ presentan necesidades más moderadas. No aparecen en la muestra participantes con un nivel de necesidades IV, que denotaría las necesidades más severas.

Por lo que se refiere a la esperada existencia de asociación entre severidad de la discapacidad y necesidades de apoyo, el contraste multivariado puso de manifiesto la existencia de diferencias significativas (Lambda de Wilks $=0.515, \mathrm{~F}(21$, $779)=9.649 ; p=0.0001)$. En la Tabla 3 se observa cómo existen diferencias significativas en todas las subescalas de la SIS. Los análisis post hoc 
revelaron que todos los grupos difieren entre sí y que quienes presentan un grado de discapacidad profunda tienen necesidades de apoyos sustancialmente más elevadas.

Por lo que se refiere al impacto de los años institucionalizados, los análisis multivariados pusieron de manifiesto la ausencia de diferencias significativas entre quienes llevaban hasta 12 años $(\mathrm{N}=133)$, de 13 a 24 años ( $N=93)$, o de 25 o más años $(\mathrm{N}=55)$ (Lambda de Wilks $=0.946, \mathrm{~F}(14,544)$ $=1.099 ; p=0.355)$.

Tabla 3.

Estadísticos descriptivos y significación de las diferencias (Anova) en la escala SIS en función de la severidad de la discapacidad - centiles

\begin{tabular}{llccccc}
\hline & & N & M & DT & F & Sig. \\
\hline VH & & & & & 49.847 & .000 \\
& Leve & 28 & 25.21 & 24.12 & & \\
moderado & 51 & 41.27 & 27.34 & & \\
& severo & 156 & 62.05 & 24.66 & & \\
& profundo & 46 & 86.52 & 13.90 & 44.836 & .000 \\
VC & & & & & & \\
& Leve & 28 & 30.61 & 26.16 & & \\
& moderado & 51 & 56.51 & 23.55 & & \\
& severo & 156 & 74.54 & 22.44 & & \\
profundo & 46 & 82.85 & 10.38 & 37.950 & .000 \\
AV & & & & & & \\
& Leve & 28 & 28.57 & 24.53 & & \\
& moderado & 51 & 54.25 & 27.21 & & \\
& severo & 156 & 70.88 & 25.58 & & \\
& profundo & 46 & 84.52 & 10.84 & 26.989 & .000 \\
AE & & & & & & \\
& Leve & 28 & 35.96 & 27.39 & & \\
& moderado & 51 & 53.39 & 27.29 & & \\
& severo & 156 & 68.46 & 25.99 & & \\
profundo & 46 & 84.02 & 10.96 & 36.879 & .000 \\
SS & & & & & & \\
& Leve & 28 & 36.25 & 27.58 & & \\
& moderado & 51 & 52.20 & 25.25 & & \\
& severo & 156 & 72.22 & 24.08 & & \\
profundo & 46 & 86.33 & 11.16 & 31.573 & .000 \\
AS & & & & & \\
& Leve & 28 & 33.32 & 24.59 & & \\
& moderado & 51 & 47.59 & 28.55 & & \\
& severo & 156 & 66.69 & 27.30 & & \\
profundo & 46 & 85.22 & 12.95 & & \\
\hline & & & & & &
\end{tabular}

Nota: $\mathrm{VH}=$ Actividades de la vida en el hogar; $\mathrm{VC}=$ Actividades de la vida en la comunidad; $\mathrm{AV}=$ Actividades de aprendizaje a lo largo de la vida; $\mathrm{AE}=$ Actividades de empleo; $\mathrm{SS}=$ Actividades de salud y seguridad; $A S=$ Actividades sociales.

De acuerdo con nuestra tercera hipótesis, predecíamos por un lado, la ausencia de diferencias significativas entre necesidades de apoyo y edad, por tratarse todos ellos de residentes mayores de 18 años. En este caso el contraste multivariado puso de manifiesto la ausencia de diferencias significativas (Lambda de Wilks $=0.045, \mathrm{~F}(12,544)$ $=0.876 \mathrm{p}=0.588$ ). Sin embargo, por lo que se refiere al género, segunda parte de nuestra predicción, los análisis multivariados evidenciaron la presencia de diferencias significativas asociadas a esta variable (Lambda de Wilks $=0.915, F(7,274)$ $=3.618 ; p=0.001)$. En la Tabla 4 se puede apreciar cómo los varones puntúan significativamente más elevado en necesidades de apoyo relacionadas con el empleo y con las actividades sociales, que sus iguales del género femenino.

Tabla 4.

Estadísticos descriptivos y significación de las diferencias (Anova) en la escala SIS en función del género - centiles

\begin{tabular}{llccccc}
\hline & & N & M & DT & F & Sig. \\
\hline VH & & & & & 1.364 & .244 \\
& $\begin{array}{l}\text { Hombre } \\
\text { Mujer }\end{array}$ & 141 & 60.46 & 27.10 & & \\
VC & 141 & 56.37 & 31.57 & & \\
& $\begin{array}{l}\text { Hombre } \\
\text { Mujer }\end{array}$ & 141 & 70.13 & 24.90 & 1.775 & .184 \\
AV & 141 & 65.95 & 27.67 & & \\
& Hombre & 141 & 68.92 & 25.58 & & \\
& Mujer & 141 & 62.43 & 30.98 & & \\
AE & & & & & 5.998 & .015 \\
& $\begin{array}{l}\text { Hombre } \\
\text { Mujer }\end{array}$ & 141 & 68.92 & 24.20 & & \\
SS & 141 & 60.87 & 30.67 & & \\
& & & & 1.505 & .221 \\
& $\begin{array}{l}\text { Hombre } \\
\text { Mujer }\end{array}$ & 141 & 69.23 & 25.21 & & \\
AS & 141 & 65.27 & 28.93 & & \\
& & & & 8.953 & .003 \\
& $\begin{array}{l}\text { Hombre } \\
\text { Mujer }\end{array}$ & 141 & 68.04 & 26.77 & & \\
\hline
\end{tabular}


Nota: $\mathrm{VH}=$ Actividades de la vida en el hogar; $\mathrm{VC}=$ Actividades de la vida en la comunidad; AV= Actividades de aprendizaje a lo largo de la vida; $\mathrm{AE}=$ Actividades de empleo; $\mathrm{SS}=$ Actividades de salud y seguridad; $A S=$ Actividades sociales.

\section{Discusión}

El presente estudio nos ha permitido demostrar la utilidad de la escala SIS para evaluar las necesidades normativas de apoyo en personas con discapacidad intelectual que se encuentran institucionalizadas. Los análisis de fiabilidad y validez arrojan resultados muy similares a los obtenidos por los autores de la adaptación española (Verdugo, et ál., 2010). Así, conforme a lo esperado, hemos obtenido niveles de fiabilidad elevados para las diferentes secciones de la escala. Por otro lado, los datos indican que las necesidades de apoyo en adultos se encuentran asociadas a la severidad de la discapacidad intelectual, pero no a la edad. En segundo lugar, y en contra de nuestras predicciones, los análisis sugieren que un sustancial porcentaje de los residentes muestra bajas necesidades de apoyo y que la población evaluada presenta un perfil bastante homogéneo. Las bajas necesidades de apoyo no se corresponden, con las oportunidades de inclusión y participación en la comunidad que se les ofrecen a los residentes. Una posible explicación es que, en el contexto chileno, la permanencia en instituciones puede ser más producto de la ausencia de alternativas de emplazamientos en la comunidad que a las extensas necesidades de apoyos de los usuarios. Si bien en Chile se están realizando esfuerzos por parte de instituciones públicas y privadas por brindar apoyos que mejoren la calidad de vida de residentes en centros permanentes (Gobierno de Chile, 2010), es preciso seguir trabajando por mejorar el capital social de los servicios ofrecidos a estas personas. Los cambios en la orientación de estos centros tendrán indudables efectos positivos en la vida de estas personas, a tenor de experiencias previas (Curran, 2008; Howe et ál., 1998; Emerson et ál., 2001; Lakin \& Stancliffe, 2007; Mansell et ál., 2006; Stancliffe et ál., 2000).

Otra posible explicación de estos resultados se relaciona con las expectativas de los profesionales sobre los usuarios. Así, aunque las instrucciones de la SIS establecen claramente la importancia de reflejar a través de las puntuaciones los apoyos que serían necesarios para que la persona tuviera éxito en cada actividad, sin tener en cuenta los servicios o apoyos proporcionados o disponibles actualmente, podría haber sucedido que los profesionales valoraran los apoyos disponibles más que los susceptibles de ser utilizados. Ello explicaría a su vez el hecho de que las puntuaciones más elevadas se obtengan en habilidades de tipo académico estrechamente relacionadas con las Actividades de aprendizaje a lo largo de la vida, mientras que las menores necesidades se obtengan en Actividades de vida en el hogar, pese a que los residentes cuentan con profesionales que desempeñan estas actividades por ellos. En la misma línea se podrían interpretar los resultados relativos al área de protección y defensa, en la que parece ponerse de manifiesto una actitud protectora o garante de aspectos básicos como la seguridad. Todo ello pone de manifiesto la importancia de hacer aflorar entre los proveedores de servicios, sus modelos mentales. El intercambio de opiniones, el análisis de la relación entre la concepción de los apoyos y la calidad de vida permitirá la puesta en marcha de cambios dentro de una cultura que promueva prácticas y políticas centradas en la mejora de dicha calidad de vida (Schalock, Bonham \& Verdugo, 2008).

El presente estudio pone también de manifiesto la existencia de una asociación entre severidad de discapacidad y necesidades de apoyo. Es preciso en este sentido subrayar que el principio de mayor necesidad no debe en cualquier caso asociarse con entornos más restrictivos (Gardner, Carran \& Nudler, 2001). Es especialmente importante fomentar la autodeterminación, la capacidad de elegir en estas personas que viven en entornos restrictivos (Lakin \& Stancliffe, 2007; Robertson et ál., 2001; Stancliffe y Parmenter, 1999).

Cabe mencionar también, cómo en nuestro estudio parece que la institucionalización juega un papel unificador en la percepción de las necesidades de apoyo de los usuarios, pese a que cabría esperar mayores necesidades en quienes llevan más años en una situación segregada. Por 
otro lado, en contra de nuestras predicciones, parece apreciarse el influjo de variables culturales asociadas al género. Así, los varones son percibidos con mayores necesidades de apoyo relacionadas con el empleo y las actividades sociales. Estas diferencias podrían estar asociadas a roles de género y a las mayores oportunidades ofrecidas a los hombres frente a las mujeres. La doble desventaja a la que se enfrentan las mujeres con discapacidad es internacionalmente reconocida (Mondéjar, Vargas, Meseguer \& Mondéjar, 2009).

En general los resultados de esta investigación deben servir para establecer nuevas prioridades por parte de las instituciones y a nivel estatal, con el fin de que las personas con discapacidad obtengan resultados deseados. Varias instituciones a nivel internacional están llevando a cabo grandes cambios en las prácticas orientadas hacia el trabajo con personas con discapacidad intelectual, a partir de la aplicación de la SIS, de las que es posible tomar ejemplo (Van Loon, 2006, van Loon et ál., 2010).

Para finalizar es preciso mencionar diferentes limitaciones del presente estudio. La primera tiene que ver con la selección de una muestra por conveniencia procedente de una única fundación. Ello limita la posibilidad de generalizar nuestros resultados. Futuras investigaciones debieran incluir una mayor diversidad de instituciones y de alternativas residenciales, para ratificar, en su caso, los resultados obtenidos. Por otro lado, en el presente estudio hemos evaluado las necesidades de apoyos normativas u objetivas como un primer paso para, en posteriores estudios, evaluar las necesidades sentidas (Thompson et ál., 2009). Ello favorecerá además el paso de una necesidad "sentida" a otra "expresada o demandada", que óptimamente se traducirá en la solicitud de servicios por parte de este colectivo.

\section{Referencias}

AAIDD- American Association on Intellectual and Developmental Disabilities (2010). Intellectual Disability. Definition, classification and systems of support. Washington,
D.C.: American Association on Intellectual and Developmental Disabilities.

AAMR- American Association on Mental Retardation (2002). Mental Retardation. Definition, classification and systems of support. Washington, D.C.: American Association on Mental Retardation.

American Psychiatric Association, A. (2003). Manual of Mental Disorders DSM-IV-TR Fourth Edition (Text Revision). Washington, D.C.: American Psychiatric Association.

Ato, M. \& Vallejo, G. (2007). Diseños experimentales en psicología. Madrid: Pirámide.

Carter, E. W., Owens, L., Trainor, A. A., Sun, Y. \& Swedeen, B. (2009). Self-determination skills and opportunities of adolescents with severe intellectual and developmental disabilities. American Journal on Intellectual and Developmental Disabilities, 114(3), 179-192.

Cooley, W. W. \& Lohnes, P. R. (1971). Multivariate data analysis. New York: John Wiley \& Sons.

Cruz, M., Jenaro, C., Pérez, M. C. \& Flores, N. (2010). Aplicabilidade da versao espanhola da escala de intensidade de apoio, na populacao mexicana com docenca mental severa. Revista Latino-Americana de Enfermagem, 18, 975-982.

Curran, J. (2008). Social Capital: Working towards a community that is open to all. Journal of Vocational Rehabilitation, 29(3), 179-183.

Emerson, E., Robertson, J., Gregory, N., Hatton, C., Kessissoglou, S., Hallam, A., Jarbrink, K., Knapp, M., Netton, A. \& Walsh P. N. (2001) Quality and costs of supported living residences and group homes in the United Kingdom. American Journal on Mental Retardation, 106, 401-415.

Gardner, J. F., Carran, D. T. \& Nudler, S. (2001). Measuring quality of life and quality of services through personal outcomes measures: 
Implications for public policy International Review of Research in Mental Retardation, 24, 75-100.

Gobierno de Chile Ley No. 20. 422 (2010). Establece normas sobre igualdad de oportunidades e inclusión social de personas con discapacidad. Gobierno de Chile (Diario Oficial, 10 de febrero, 2010).

Howe, J., Horner, R. \& Newton, J. (1998). Comparison of supported living and traditional residential services in the state Oregon. Mental Retardation, 36, 1-11.

Jenaro, C., Cruz, M., Pérez, M. C., Flores, N. \& Vega, V. (2011). Utilization of the supports intensity scale with psychiatric populations: psychometric properties and utility for service delivery planning. Archives of Psychiatric Nursing, 25(5), e9-e17. doi: http://dx.doi. org/10.1016/j.apnu.2011.05.002.

Lakin, C. \& Stancliffe, R. (2007). Residential supports for persons with intellectual and Developmental disabilities. Mental Retardation and Developmental Disabilities Research Reviews, (13), 151-159.

Luckasson, R., Coulter, D., Polloway, E., Reiss, S., Schalock, R., Snell, M., Spitalnik, D. \& Stark, J. (1992). Mental retardation: Classification and systems of supports (9th ed.). Washington, D.C.: American Association on Mental Retardation.

Mansell, J. (2006). Deinstitutionalisation and community living: Progress, problems and Priorities, Journal of Intellectual \& Developmental Disability, 31(2), 65-76.

Mansell, J., Beadle-Brown, J., Skidmore, C., Whelton, B. \& Hutchinson, A. (2006). People with learning disabilities in 'out-of-area' residential placements: 1. Policy context Journal of Intellectual Disability Research, 50(II), 837-844.
McCormack, B. \& Farrell, M. (2009). Translating quality of life into service action: Use of personal outcome measures in the Republic of Ireland. British Journal of Learning Disabilities, 37, 300-307.

Mondéjar, J., Vargas, M., Meseguer, M. L. \& Mondéjar, J. A. (2009). Impact of social factors on labour discrimination of disabled women. Research in Developmental Disabilities, 30(6), 1115-1123.

Mora, Córdoba, Bedoya, Verdugo (2007). Características de la calidad de vida de las familias con un adulto con discapacidad intelectual (DI)/RM, en la ciudad de Cali, Colombia. Revista Diversitas, 3(1), 37-54.

Robertson, J., Emerson, E., Hatton, C., Gregory, N., Kessissoglou, S., Hallam, A., et ál., (2001). Environmental opportunities and supports for exercising self-determination in community-based residential settings. Research in Developmental Disabilities, 22, 487-502.

Schalock, R. L., Bonham, G. S. \& Verdugo, M. A. (2008). The conceptualization and measurement of quality of life: Implications for program planning and evaluation in the field of intellectual disabilities. Evaluation and Program Planning, 31(2), 181-190.

Stancliffe, R. \& Parmenter, T. R. (1999). The choice questionnaire: A scale to assess choices exercised by adults with intellectual disability. Journal of Intellectual and Developmental Disability, 24, 107-132.

Stancliffe, R., Emerson, E. \& Lakin, C. (2000). Community living and people with intellectual disability: Introduction to Part I Journal of Intellectual and Developmental Disability, $25,1-4$

Thompson, J. R., Bradley, V. J., Buntinx, W. H., Schalock, R. L., Shogren, K. A., Snell, M. E. et ál., (2009). Conceptualizing supports and the support needs of people with intellectual 
disability. Intellectual and Developmental Disabilities, 47(2), 135-146.

Thompson, J. R., Bryant, B. R., Campbell, E. M., Craig. E.M., Hughes, C. M., Rotholz, D. A. et ál., (2004). Supports Intensity Scale user's manual. Washington, DC: American Association on Mental Retardation.

Van Loon, J. (2006). Los apoyos en Arduin. Sobre las diversas facetas de los apoyos y la implementación de la escala de intensidad de apoyos (SIS) en una organización de servicios. Siglo Cero, 37(4), 17-34.

Van Loon, J., Claes, C., Vandevelde, S., Van Hove, G. \& Schalock, R. L. (2010). Assessing individual support needs to enhance personal outcomes. Exceptionality, 18(4), 193-202.
Vega, V., Jenaro, C., Morillo, M. L., Cruz, M. \& Flores, N. (2011). Calidad de vida y apoyos en personas con discapacidad intelectual institucionalizados en Chile: estudio piloto. Psicología, Conocimiento y Sociedad, 3, 52-70.

Verdugo, M. A., Arias, B. \& Ibáñez, A. (2007). SIS. Escala de Intensidad de Apoyos. Manual. Adaptación española. Madrid: TEA.

Verdugo, M. A., Arias, B., Ibañez, A. \& Schalock, R. L. (2010). Adaptation and psychometric properties of the Spanish version of the Supports Intensity Scale (SIS). American Journal on Intellectual and Developmental Disabilities, 115(6), 496-503. 